\title{
Perturbation of a Globally Stable Steady State and Uniform Persistence
}

\author{
Pierre Magal
}

Received: 7 January 2005 / Revised: 10 August 2008 / Published online: 2 December 2008

(C) Springer Science+Business Media, LLC 2008

\begin{abstract}
In this paper we consider a parametrized family of semi-flows with continuous or discrete time. In the spirit of the global stability result proved by Smith and Waltman (Proc AMS 127:447-453, 1999) we use the upper semi-continuity of a parametrized family of global attractors. Here we investigate the case where the linearized equation of the unperturbed system has a simple dominant eigenvalue 0 in the case of a continuous time system (or 1 in the case of a discrete time system). New difficulties arise since such a system may exhibit a bifurcation. The goal of the paper is to describe the global dynamics of the perturbed system.
\end{abstract}

Keywords Global stability · Uniform persistence · Global attractors · Upper semi-continuity

\section{AMS Subject Classifications $\quad 37 \mathrm{C} 25 \cdot 37 \mathrm{C} 70 \cdot 37 \mathrm{~L} 05 \cdot 37 \mathrm{~N} 25$}

\section{Introduction}

Uniform persistence is an important concept in population dynamics since it characterizes the long-term survival of some or all interacting species in an ecosystem. There have been extensive investigations on uniform persistence for discrete and continuous-time dynamical systems. We refer to $[8,22,47,55]$ for surveys and reviews. Roughly speaking, uniform persistence is the notion saying that a closed subset of the state space (e.g., the set of extinction for one or more populations) is repelling for the dynamics on the complementary set. A natural question is about the existence of "interior" global attractors and "coexistence" steady states for uniformly persistent dynamical systems. The existence of interior global attractors was addressed by Hale and Waltman [19], and the existence of coexistence steady states under a general setting was investigated by Zhao [54] and Magal and Zhao [36]. In [19,54] the

P. Magal $(\varangle)$

Faculte des Sciences et Techniques, B. P. 540, 76058 Le Havre, France

e-mail: pierre.magal@univ-lehavre.fr 
classical concept of global attractors was employed: a global attractor is a compact, invariant set which attracts every bounded set in the phase space (see, e.g., Hale [11], Temam [44], Cholewa and Dlotko [5], Raugel [38]). Recently, a weaker concept of global attractors was introduced by Hirsch et al. [21] and Sell and You [39]: a global attractor is a compact, invariant set which attracts some neighborhood of itself and every point in the phase space. In Magal and Zhao [36], several examples were considered in order to discuss the properties of global attractors in the context of uniform persistence (see also Cholewa and Hale [6]). These examples show that the notion of global attractor of $[21,39]$ is more appropriate in the context of uniform persistence. For this reason, we will use this non-classical definition here. We want to point out that this more recent notion of global attractor is not classical and does not coincide with the one used by Hale [11]. We also want to notice that, when the semi-flow is asymptotically smooth, the notion of global attractor used in this paper here coincides with the notion of a compact invariant set attracting all the compact sets.

In what follows, we will make the following assumption.

Assumption A Let M be a closed subset of a Banach space $(X,\|\cdot\|)$, let $\left(\Lambda, d_{\Lambda}\right)$ be a metric space, and let $\widehat{\lambda} \in \Lambda$ be fixed. Let $\left\{U_{\lambda}(t)\right\}_{t \in I}$ (with $I=\mathbb{R}$ or $I=\omega \mathbb{N}$ for some $\omega>0$ ) be a family of asymptotically smooth continuous semi-flows on M parametrized by $\lambda \in \Lambda$. We assume that the map $(t, \lambda, x) \rightarrow U_{\lambda}(t) x$ is continuous from $I \times \Lambda \times M$ into M. Let $\rho: \Lambda \times M \rightarrow[0,+\infty)$ be a continuous map. For each $\lambda \in \Lambda$, we set

$$
\partial M_{0}^{\lambda}=\left\{x \in M: \rho_{\lambda}(x)=0\right\} \text { and } M_{0}^{\lambda}=\left\{x \in M: \rho_{\lambda}(x)>0\right\} .
$$

We assume that $M_{0}^{\lambda}$ is positively invariant by $U_{\lambda}$. We also assume that:

(a) For each $\lambda \in \Lambda, U_{\lambda}$ has a global attractor $A_{\lambda}$ in $(M, d)$.

(b) The family $\left\{A_{\lambda}\right\}_{\lambda \in \Lambda}$ is upper semi-continuous at $\widehat{\lambda} \in \Lambda$.

(c) For each $\lambda \in \Lambda, U_{\lambda}$ is $\rho_{\lambda}$-uniformly persistent, that is, for each $\lambda \in \Lambda$, there exists $\varepsilon_{\lambda}>0$, such that

$$
\liminf _{t \rightarrow+\infty} \rho_{\lambda}\left(U_{\lambda}(t) x\right) \geq \varepsilon_{\lambda}, \quad \forall x \in M_{0}^{\lambda} .
$$

We recall that the family $\left\{A_{\lambda}\right\}_{\lambda \in \Lambda}$ of subsets of $M$ is upper semi-continuous at $\widehat{\lambda}$, if

$$
\delta\left(A_{\lambda}, A_{\widehat{\lambda}}\right) \rightarrow 0 \text { as } \lambda \rightarrow \widehat{\lambda} .
$$

where

$$
\delta(B, A):=\sup _{x \in B} d(x, A), \text { and } d(x, A):=\inf _{y \in A} d(x, y) .
$$

The property of upper semi-continuity of a familly of global attractor has been investigated in many contexts and we refer to $[4,11,12,15-18,20,26,37-39,43,51]$ for a nice survey on the subject. In the context of uniform persistence we have the following result.

Theorem 1.1 Let Assumption A be satisfied. Then for each $\lambda \in \Lambda, U_{\lambda}$ has a global attractor $A_{0 \lambda}$ in $M_{0}^{\lambda}$. Moreover the family $\left\{A_{0 \lambda}\right\}_{\lambda \in \Lambda}$ is upper semi-continuous at $\widehat{\lambda}$ if and only if there exist $\varepsilon>0$, and $\delta>0$, such that

$$
\limsup _{t \rightarrow+\infty} \rho_{\lambda}\left(U_{\lambda}(t) x\right) \geq \varepsilon, \quad \forall x \in M_{0}^{\lambda}, \forall \lambda \in \bar{B}_{\Lambda}(\widehat{\lambda}, \delta),
$$

where $\bar{B}_{\Lambda}(\widehat{\lambda}, \delta):=\left\{\lambda \in \Lambda: d_{\Lambda}(\widehat{\lambda}, \lambda) \leq \delta\right\}$. 
In order to study the local pertubation problem we make the following assumption.

Assumption B We assume that there exists a globally asymptotically stable equilibrium point $\bar{x}_{\widehat{\lambda}}$ for $U_{\widehat{\lambda}}$ in $M_{0}^{\widehat{\lambda}}\left(\right.$ i.e. $\left.A_{0 \widehat{\lambda}}=\left\{\bar{x}_{\widehat{\lambda}}\right\}\right)$.

Smith and Waltman [42] prove that, when $M_{0}^{\lambda}=M$ for each $\lambda \in \Lambda$, and $\bar{x}_{\widehat{\lambda}}$ is locally exponentially stable, then the perturbed system has also a globally asymptotically stable equilibrium. Motivated by some examples in population dynamics (see for example Magal and Webb [35]), we generalize the result of Smith and Waltman [42] to the context of uniform persistence.

Theorem 1.2 Let Assumptions A and B be satisfied, and assume in addition that:

(a) The family $\left\{A_{0 \lambda}\right\}_{\lambda \in \Lambda}$ is upper semi-continuous at $\widehat{\lambda}$.

(b) There exist $t_{0} \in I \backslash\{0\}$ and a bounded linear operator $L: X \rightarrow X$ such that the spectral radius $r(L)$ of $L$ satisfies $r(L)<1$ and such that

$$
\lim _{\delta \rightarrow 0} \sup _{\left.\lambda \in \bar{B}_{\Lambda} \widehat{\lambda}, \delta\right)}\left\|U_{\lambda}\left(t_{0}\right)-L\right\|_{L i p, \bar{B}_{M}\left(\bar{x}_{\widehat{\lambda}}, \delta\right)}=0 .
$$

Then there exist $\delta>0$, and, for for each $\lambda \in \bar{B}_{\Lambda}(\widehat{\lambda}, \delta)$, a globally asymptotically stable equilibrium point $\bar{x}_{\lambda}$ for $U_{\lambda}$ in $M_{0}^{\lambda}$.

(For the definition of the norm $\|\cdot\|_{L i p, \bar{B}_{M}\left(\bar{x}_{\widehat{\lambda}}, \delta\right)}$, we refer the reader to the beginning of Sect. 3)

Remark 1.3 In practice for continuous-time dynamical systems, Assumption(B) in Theorem 1.2 will be satisfied if we can find a linear $C_{0}$-semi-group $\{V(t)\}_{t \in I}$ on $X$ with negative growth rate $\left(\right.$ i.e. $\left.\lim _{t \rightarrow+\infty} \frac{\ln \left(\|V(t)\|_{\mathcal{L}(X)}\right)}{t}<0\right)$ such that

$$
\lim _{\delta \rightarrow 0} \sup _{\lambda \in \bar{B}_{\Lambda}(\widehat{\lambda}, \delta)}\left\|U_{\lambda}(t)-V(t)\right\|_{L i p, \bar{B}_{M}\left(\bar{x}_{\widehat{\lambda}}, \delta\right)}=0, \quad \forall t \in I .
$$

The main goal of this paper is to describe the asymptotic behavior of the perturbed system $U_{\lambda}(t)$ whenever 1 is a simple dominant eigenvalue of the linear operator $L$ (or 0 is a simple dominant eigenvalue of the infinitesimal generator of $\{V(t)\}_{t \in I}$ ). In this case the parametrized system may exhibit a bifurcation at $\lambda=\widehat{\lambda}$ (see examples in Sect. 4). This type of assumption is relatively natural in the context of population dynamics, because this property is usually associated to the positivity of the linear operator $L$ (or the positivity of the linear $C_{0}$-semigroups $\left.\{V(t)\}_{t \in I}\right)$. For example, assume that $(X,\|\|$.$) is a Banach lattice for some partial$ order $\leq$ on $X$; if $\{V(t)\}_{t \in I}$ is irreducible, then it is known that there is a simple dominant eigenvalue of the infinitesimal generator of $\{V(t)\}_{t \in I}$ ( see [53]). We also refer to [3,10,46] for a nice survey on this subject.

Let $K$ be a subset of $X$. A point $x \in K$ is said to be extreme in $K$ (in the sense of Minkowski) if $x=\lambda y+(1-\lambda) z$ with $y, z \in K$, and $\lambda \in(0,1)$ implies $x=y=z$.

The main result of this paper is the following theorem.

Theorem 1.4 Let Assumptions A and B be satisfied. We assume in addition that:

(a) For each $\lambda \in \Lambda, A_{0 \lambda}$ is connected.

(b) The family $\left\{A_{0 \lambda}\right\}_{\lambda \in \Lambda}$ is upper semi-continuous at $\widehat{\lambda}$. 
(c) There exist $t_{0} \in I \backslash\{0\}$, a bounded linear operator $L: X \rightarrow X$ and a bounded linear projector $P: X \rightarrow X$ with rank 1, such that

$$
L P=P L=P \text { and } r(L(I-P))<1,
$$

and

$$
\lim _{\delta \rightarrow 0} \sup _{\left.\lambda \in \bar{B}_{\Lambda} \widehat{\lambda}, \delta\right)}\left\|U_{\lambda}\left(t_{0}\right)-L\right\|_{L i p, \bar{B}_{M}\left(\overline{x_{\widehat{\lambda}}}, \delta\right)}=0 .
$$

(d) For each $\lambda \in \Lambda \backslash\{\widehat{\lambda}\}$, one of the following conditions is satisfied:

(1) There exists an equilibrium point $\bar{x}_{\lambda}$ of $U_{\lambda}$ in $M_{0}^{\lambda}$, such that $P\left(\bar{x}_{\lambda}\right)$ is extreme in $P\left(A_{0 \lambda}\right)$ or in $P\left(M_{0}^{\lambda}\right)$.

(2) There exist two disjoint non-empty compact connected subsets $E_{1 \lambda}, E_{2 \lambda} \subset M_{0}^{\lambda}$ which are positively invariant by $U_{\lambda}$.

Then there exists $\delta>0$, such that for each $\lambda \in \bar{B}_{\Lambda}(\widehat{\lambda}, \delta)$, the following assertions are satisfied:

(i) There exists a map $\phi_{\lambda} \in \operatorname{Lip}\left(P\left(A_{0 \lambda}\right),(I d-P)\left(A_{0 \lambda}\right)\right)$ such that

$$
A_{0 \lambda}=\left\{y+\phi_{\lambda}(y): y \in P\left(A_{0 \lambda}\right)\right\} .
$$

(ii) For each $t>0, U_{\lambda}(t)$ is a bijection from $A_{0 \lambda}$ into itself. Furthermore, if we set $U_{\lambda}(-t)=U_{\lambda}(t)^{-1}, \forall t \in I$, then $\left\{U_{\lambda}(t)\right\}_{t \in I}$ is a strongly continuous group on $A_{0 \lambda}$. Moreover, for each $x \in A_{0 \lambda}$, there exist two equilibrium points $\bar{x}_{+}, \bar{x}_{-} \in$ $A_{0 \lambda}$ of $\left\{U_{\lambda}(t)\right\}_{t \in I}$, such that

$$
U_{\lambda}(t) \rightarrow \bar{x}_{+} \text {and } U_{\lambda}(-t)(x) \rightarrow \bar{x}_{-} \text {as } t \rightarrow+\infty .
$$

(iii) If $M_{0}^{\lambda}$ contains at most a finite number of equilibria for $\left\{U_{\lambda}(t)\right\}_{t \in I}$, then, for each $x \in M_{0}^{\lambda}$, there exists an equilibrium $\bar{x} \in A_{0 \lambda}$ of $U_{\lambda}(t)$, such that

$$
U_{\lambda}(t)(x) \rightarrow \bar{x} \text {, as } t \rightarrow+\infty \text {. }
$$

(iv) If there exists a compact subset $B_{\lambda} \subset M_{0}^{\lambda}$ which is invariant by $U_{\lambda}$ and contains at most one equilibrium of $U_{\lambda}$, then $B_{\lambda}=\left\{\bar{x}_{\lambda}\right\}$, and $\bar{x}_{\lambda}$ is an equilibrium of $U_{\lambda}$.

(v) We set

$$
T(\lambda, x)=U_{\lambda}\left(t_{0}\right) x, \quad \forall(\lambda, x) \in \Lambda \times M .
$$

Assume in addition that there exist a neighborhood $V$ of $\left(\widehat{\lambda}, \bar{x}_{\widehat{\lambda}}\right)$ in $\Lambda \times X$ and an extension $\widehat{T}$ of $T$ to $V$, such that

$$
\lim _{\delta \rightarrow 0} \sup _{\lambda \in \Lambda: d_{\Lambda}(\lambda, \widehat{\lambda}) \leq \delta}\left\|\widehat{T}_{\lambda}-L\right\|_{L i p, \bar{B}_{X}\left(\bar{x}_{\widehat{\lambda}}, \delta\right)}=0 .
$$

Then there exist $\delta^{*} \in(0, \delta)$ and, for each $\lambda \in \bar{B}_{\Lambda}\left(\widehat{\lambda}, \delta^{*}\right)$ and each $x \in M_{0}^{\lambda}$, an equilibrium point $\bar{x}=\bar{x}(\lambda, x) \in A_{0 \lambda}$ of $\left\{U_{\lambda}(t)\right\}_{t \in I}$, such that

$$
U_{\lambda}(t) x \rightarrow \bar{x} \text { as } t \rightarrow+\infty .
$$

In Assumption A, the subset $\partial M_{0}^{\lambda}$ can be empty for each $\lambda \in \Lambda$. In this case, we have $M_{0}^{\lambda}=M$, and $A_{\lambda}=A_{0 \lambda}$, and Theorem 1.2 corresponds to the result of Smith and Waltman [42]. One may also apply Theorem 1.4 on $M$ by considering the case where $\partial M_{0}^{\lambda}$ is empty. Theorem 1.4 generalizes the results obtained in $[29,30]$ for discrete time dynamical system. 
The plan of the paper is the following. In Sect.2, we recall some results about global attractors, and we investigate upper semi-continuity results in the context of uniform persistence. In Sect. 3, we prove Theorems 1.2 and 1.4. In Sect. 4, we apply Theorem 1.4 to some examples of age-structured population dynamics models.

\section{Attractors}

Let $(M, d)$ be a complete metric space. For any subsets $A, B \subset M$ and any $\epsilon>0$, we define

$$
\begin{aligned}
& d_{H}(A, B)=\max (\delta(B, A), \delta(A, B)) \text { (Hausdorff's metric) } \\
& N(A, \epsilon):=\{x \in M: d(x, A)<\epsilon\}, \bar{N}(A, \epsilon):=\{x \in M: d(x, A) \leq \epsilon\}, \\
& B(y, \epsilon):=N(\{y\}, \epsilon), \text { and } \bar{B}(A, \epsilon):=\bar{N}(\{y\}, \epsilon) .
\end{aligned}
$$

Let $I$ be a subset of $\mathbb{R}_{+}$, which is either 1) $I=\omega \mathbb{N}$ for some $\omega \in \mathbb{R}_{+} \backslash\{0\}$; or 2) $I=\mathbb{R}_{+}$. Let $\{U(t)\}_{t \in I}$ be a family of maps from $M$ into itself. We say that $\{U(t)\}_{t \in I}$ is a semi-flow if

$$
U(0)=I d \text { and } U(t+s)=U(t) \circ U(s), \quad \forall t, s \in I .
$$

We will say that $U$ is continuous if the map $(t, x) \rightarrow U(t) x$ is a continuous map from $I \times M$ into $M$. Here the case $I=\mathbb{R}_{+}$or (respectively $I=\omega \mathbb{N}$ for some $\omega \in \mathbb{R}_{+} \backslash\{0\}$ ) corresponds to the case of continuous (respectively discrete) time semi-flows.

When $I=\omega \mathbb{N}$, we set $T=U(\omega)$. Then, $U(n \omega)=T^{n}$, where $T^{0}=I d$ and $T^{n}=$ $T \circ T^{n-1}, \forall n \geq 1$. Moreover in the discrete time case, $U$ is continuous if and only if $T=U(\omega)$ is a continuous map.

For each subset $B \subset M$, we denote by $\gamma^{+}(B)=\bigcup_{t \geq 0} U(t)(B)$ the positive orbit of $B$ for $U$, and

$$
\omega(B)=\bigcap_{t \geq 0} \overline{\bigcup_{s \geq t} U(s)(B)}
$$

the omega-limit set of $B$. A subset $A \subset M$ is positively invariant for $U$ if $U(t)(A) \subset A, \forall t \geq$ 0 . $A$ is invariant for $U$ if $U(t)(A)=A, \forall t \geq 0$. We say that a subset $A \subset M$ attracts a subset $B \subset M$ for $U$, if $\lim _{t \rightarrow \infty} \delta(U(t) B, A)=0$.

To give a unified definition of internally transitive sets for both discrete and continuous time dynamical systems, we set

$$
\sigma_{t}= \begin{cases}t, & \text { if } I=\mathbb{R}, \\ \omega, & \text { if } I=\omega \mathbb{N} \text { (for some } \omega>0),\end{cases}
$$

whenever $t \in I \backslash\{0\}$. Let $A$ be a subset of $M$, and let $a, b \in A$. We say that $a$ is chained to $b$ in $A$ if for each $t \in I \backslash\{0\}$, each $\varepsilon>0$, and each $\eta>0$, there exist $\tau \in\left[\sigma_{t}, \sigma_{t}+\eta\right] \cap I$ and $x_{1}, x_{2}, \ldots, x_{m} \in A$ (with $m \geq 2$ ) such that

$$
x_{1}=a, x_{m}=b, \text { and } d\left(U(\tau) x_{i}, x_{i+1}\right) \leq \varepsilon, \quad \forall i=1, \ldots, m-1 .
$$

A set $A$ is said to be internally chain transitive if for each pair $a, b \in A, a$ is chained to $b$ in $A$.

In the discrete time case the previous definition coincides with the one in ([21], Sect. 2), but in the continuous time case, our definition is different from the definition in ([21], Sect.2). Note that, the above notion of internally chain transitive set will be used in the proof of Lemma 3.3. 
Following LaSalle [25], a compact invariant subset is said to be invariantly connected if it cannot be decomposed into two disjoint closed non-empty invariant subsets. It is easy to see that every internally chain transitive set is invariantly connected.

Following the arguments used in ([21], Lemma 2.1) we obtain the following lemma.

Lemma 2.1 Let $\{U(t)\}_{t \in I}$ be a continuous semi-flow on $(M, d)$. Then the omega (alpha) limit set of a relatively compact positive (negative) orbit of a point is internally chain transitive.

The first main tool used in investigating the existence of global attractors is the following result. Its proof follows the same lines as the one in ([36], Lemma 2.2).

Lemma 2.2 Let $\{U(t)\}_{t \in I}$ be a continuous semi-flow. Let $A$ be a subset of $M$, and assume that there exists a compact subset $C \subset M$, which attracts $A$ for $U$. Then $\omega(A)$ is non-empty, compact, invariant for $U$, and attracts $A$.

A positively invariant subset $A \subset M$ for $U$ is said to be stable if for any neighborhood $V$ of $A$, there exists a neighborhood $W \subset V$ of $A$ such that $U(t)(W) \subset V, \forall t \geq 0$. We say that $A$ is globally asymptotically stable for $U$ if, in addition, $A$ attracts every point in $M$ for $U$. Note that $A$ is stable if and only if for each neighborhood $V$, there exists a neighborhood $W$ of $A$ which is positively invariant by $U$ and satisfies $W \subset V$.

The second main tool used in showing the existence of global attractors is the following lemma. The proof follows the arguments used in ([11], Theorem 2.2.5).

Lemma 2.3 Let $\{U(t)\}_{t \in I}$ be a continuous semi-flow. Let $A \subset M$ be compact and positively invariant for $U$. If $A$ attracts all the compact subsets of one of its neighborhoods, then $A$ is stable.

Definition 2.4 A non-empty, compact and invariant set $A \subset M$ is said to be an attractor for $U$ if $A$ attracts one of its neighborhoods; $A$ is a global attractor for $U$ if $A$ is an attractor that attracts each point of $M$.

Definition 2.5 We say that $U$ is point dissipative if there exists a bounded set $B \subset M$ such that for each $x \in M$, there exists $t \in I$, such that $U(s) x \in B$ for each $s \geq t$. We say that $U$ is asymptotically smooth if every positively invariant bounded set is attracted by a compact subset.

The following theorem is known (see Theorem 3.2, [13]) and (Theorem 2.6, [36]) for discrete and continuous time semi-flows. The proof of this result uses similar arguments as in (Theorem 2.6, [36]) combined with Lemmas 2.2 and 2.3.

Theorem 2.6 Let $U$ be a continuous semi-flow on a complete metric space $(M, d)$. Assume that

(a) U is point dissipative and asymptotically smooth;

(b) For each compact subset $C$ of $M$, there exists $t_{C} \in I$, such that the orbit $\gamma^{+}\left(U\left(t_{C}\right)(C)\right)$ is bounded.

Then $U$ has a global attractor $A \subset M$. Moreover, for each subset $B$ of $M$, if there exists $t_{B} \geq 0$ such that $\gamma^{+}\left(U\left(t_{B}\right)(B)\right)$ is bounded, then A attracts $B$ for $U$. 
We now investigate the dependence with respect to a parameter. We refer to $[11,38,39]$ for a nice survey on this subject. Let $\left(\Lambda, d_{\Lambda}\right)$ be a complete metric space, let $\left\{U_{\lambda}(t)\right\}_{t \in I}$ be a family of semi-flows on $M$ parameterized by $\lambda \in \Lambda$. Let $\left\{A_{\lambda}\right\}_{\lambda \in \Lambda}$ be a family of compact subsets of $M$. We first have the following result.

Proposition 2.7 We assume that, for each $t \in I$, the map $(\lambda, x) \rightarrow U_{\lambda}(t) x$ is continuous, that, for each $\lambda \in \Lambda, A_{\lambda}$ is invariant by $U_{\lambda}$, and that $A_{\widehat{\lambda}}$ is a global attractor for $U_{\widehat{\lambda}}$. Then the following statements are equivalent:

(1) $\left\{A_{\lambda}\right\}_{\lambda \in \Lambda}$ is upper semi-continuous at $\widehat{\lambda} \in \Lambda$.

(2) For each sequence $\left\{\lambda_{n}\right\}_{n \geq 0}$ such that $\lambda_{n} \rightarrow \widehat{\lambda}$ as $n \rightarrow+\infty$, there exists a compact subset $C \subset M$, such that $\delta\left(A_{\lambda_{n}}, C\right) \rightarrow 0$ as $n \rightarrow+\infty$.

Proof (1) $\Rightarrow$ (2) is obvious with $C=A_{\widehat{\lambda}}$. We now prove (2) $\Rightarrow$ (1). Assume that $\left\{A_{\lambda}\right\}_{\lambda \in \Lambda}$ is not upper semi-continuous at $\widehat{\lambda} \in \Lambda$. Then we can find $\varepsilon>0$, and a sequence $\left\{\lambda_{n}\right\}_{n \geq 0} \rightarrow \widehat{\lambda}$, such that

$$
\delta\left(A_{\lambda_{n}}, A_{\widehat{\lambda}}\right) \geq \varepsilon, \quad \forall n \geq 0 .
$$

Then one can find a subsequence $\left\{\lambda_{n_{p}}\right\}_{p \geq 0}$ and a compact subset $\widehat{A}_{\infty}$ such that $d_{H}\left(A_{\lambda_{n_{p}}}, \widehat{A}_{\infty}\right) \rightarrow 0$ as $p \rightarrow+\infty$. By construction, $\delta\left(\widehat{A}_{\infty}, A_{\widehat{\lambda}}\right) \geq \varepsilon$. But for each $p \geq 0$, $A_{\lambda_{n p}}$ is invariant by $U_{\lambda_{n_{p}}}$, and for each $t \in I$, the map $(\lambda, x) \rightarrow U_{\lambda}(t) x$ is continuous, so $\widehat{A}_{\infty}$ is invariant by $U_{\widehat{\lambda}}$. Finally, since $A_{\widehat{\lambda}}$ is a global attractor for $U_{\widehat{\lambda}}$, we deduce that $\widehat{A}_{\infty} \subset A_{\widehat{\lambda}}$ and $\delta\left(\widehat{A}_{\infty}, A_{\widehat{\lambda}}\right)=0$, which leads to a contradiction.

As an immediate consequence of the previous result we have the following proposition.

Proposition 2.8 We assume that, for each $\lambda \in \Lambda, A_{\lambda}$ is invariant by $U_{\lambda}$, and that there exists a subset $B$ of $M$ such that:

(a) $A_{\widehat{\lambda}}$ attracts $B$ for $U_{\widehat{\lambda}}$.

(b) $A_{\lambda} \subset B, \forall \lambda \in \Lambda$.

(c) For each $t \in I, U_{\lambda}(t) x \rightarrow U_{\widehat{\lambda}}(t) x$ as $\lambda \rightarrow \widehat{\lambda}$ uniformly in $x \in B$.

Then $\left\{A_{\lambda}\right\}_{\lambda \in \Lambda}$ is upper semi-continuous at $\widehat{\lambda}$.

Proof The proof is straightforward.

In the application one may use the following result. This theorem is known in the context of global attractors, and for completeness we prove it.

Proposition 2.9 Let $\left(\Lambda, d_{\Lambda}\right)$ be a metric space, let $\widehat{\lambda} \in \Lambda$ be fixed, and let $\left\{U_{\lambda}(t)\right\}_{t \in I}$ be a family of continuous semi-flows on a metric space $(M, d)$ parameterized by $\lambda \in \Lambda$. We assume that:

(a) $U_{\lambda}$ is asymptotically smooth for each $\lambda \in \Lambda$.

(b) $U_{\widehat{\lambda}}$ has a global attractor $A_{\widehat{\lambda}}$.

(c) There exists a bounded subset $B_{0}$ of $M$, such that $A_{\widehat{\lambda}}$ attracts $B_{0}$ for $U_{\widehat{\lambda}}$, and, for each $\lambda \in \Lambda$ and each $x \in M$, there exists $t_{0}=t_{0}(\lambda, x) \geq 0$, such that

$$
U_{\lambda}(t) x \in B_{0}, \quad \forall t \geq t_{0} .
$$


(d) For each bounded set $B \subset M$ and each $\tau>0, U_{\lambda}(t) x \rightarrow U_{\widehat{\lambda}}(t) x$ as $\lambda \rightarrow \widehat{\lambda}$ uniformly in $(t, x) \in([0, \tau] \cap I) \times B$.

Then there exists $\eta>0$, such that for all $\lambda \in \bar{B}_{\Lambda}(\widehat{\lambda}, \eta), U_{\lambda}(t)$ has a global attractor $A_{\lambda} \subset M$, and the family $\left\{A_{\lambda}\right\}_{\lambda \in \bar{B}_{\Lambda}(\widehat{\lambda}, \eta)}$ is upper semi-continuous at $\widehat{\lambda}$.

Proof For each $\lambda \in \Lambda$, we set

$$
J_{\lambda}\left(B_{0}\right):=\left\{y \in B_{0}: U_{\lambda}(t)(y) \in B_{0}, \forall t \in I\right\} .
$$

Then $J_{\lambda}\left(B_{0}\right) \subset B_{0}$ is bounded and positively invariant by $U_{\lambda}$, and for each $x \in M$, there exists $t \geq 0$, such that $U_{\lambda}(t)(x) \in J_{\lambda}\left(B_{0}\right)$. Since $U_{\lambda}$ is asymptotically smooth, we deduce that $\omega_{\lambda}\left(\bar{J}_{\lambda}\left(B_{0}\right)\right)=\bigcap_{s \geq 0} \bigcup_{t \geq s} U_{\lambda}(t)\left(J_{\lambda}\left(B_{0}\right)\right)$ is compact, invariant by $U_{\lambda}$, and attracts the points of $M$ for $U_{\lambda}$. By applying Proposition 2.8 (with $A_{\lambda}=\omega_{\lambda}\left(J_{\lambda}\left(B_{0}\right)\right)$, whenever $\lambda \neq \widehat{\lambda}$, and $B=B_{0}$ ), we deduce that

$$
\lim _{\lambda \rightarrow \widehat{\lambda}} \delta\left(\omega_{\lambda}\left(J_{\lambda}\left(B_{0}\right)\right), A_{\widehat{\lambda}}\right)=0 .
$$

Let $\varepsilon>0$ be fixed. Since $A_{\widehat{\lambda}}$ is a global attractor for $U_{\widehat{\lambda}}$, it follows that $A_{\widehat{\lambda}}$ is stable for $U_{\widehat{\lambda}}$ and $A_{\widehat{\lambda}}$ attracts one of its neighborhoods for $U_{\widehat{\lambda}}$. So there exist $\widehat{\varepsilon} \in(0, \varepsilon)$ and $t^{*} \in I$, such that $A_{\widehat{\lambda}}$ attracts $\bar{N}\left(A_{\widehat{\lambda}}, \widehat{\varepsilon}\right)$ for $U_{\widehat{\lambda}}$,

$$
U_{\widehat{\lambda}}(t) \bar{N}\left(A_{\widehat{\lambda}}, \widehat{\varepsilon}\right) \subset \bar{N}\left(A_{\widehat{\lambda}}, \varepsilon / 4\right), \quad \forall t \geq 0,
$$

and

$$
U_{\widehat{\lambda}}\left(t^{*}\right) \bar{N}\left(A_{\widehat{\lambda}}, \widehat{\varepsilon}\right) \subset \bar{N}\left(A_{\widehat{\lambda}}, \widehat{\varepsilon} / 4\right) .
$$

By using Assumption $\mathrm{D}$, we deduce that there exists $\eta_{1}>0$, such that for each $\lambda \in \Lambda$ with $d_{\Lambda}(\widehat{\lambda}, \lambda) \leq \eta_{1}$

$$
U_{\lambda}(t) \bar{N}\left(A_{\widehat{\lambda}}, \widehat{\varepsilon}\right) \subset \bar{N}\left(A_{\widehat{\lambda}}, \varepsilon / 2\right), \quad \forall t \in\left[0, t^{*}\right] \cap I,
$$

and

$$
U_{\lambda}\left(t^{*}\right) \bar{N}\left(A_{\widehat{\lambda}}, \widehat{\varepsilon}\right) \subset \bar{N}\left(A_{\widehat{\lambda}}, \widehat{\varepsilon} / 2\right) .
$$

We set, for each $\lambda \in \Lambda$, with $d_{\Lambda}(\widehat{\lambda}, \lambda) \leq \eta_{1}$,

$$
B_{\lambda}=\overline{\bigcup_{t \in\left[0, t^{*}\right]} U_{\lambda}(t) \bar{N}\left(A_{\widehat{\lambda}}, \widehat{\varepsilon}\right)},
$$

then $B_{\lambda}$ is positively invariant by $U_{\lambda}$ and $\bar{N}\left(A_{\widehat{\lambda}}, \widehat{\varepsilon}\right) \subset B_{\lambda} \subset \bar{N}\left(A_{\widehat{\lambda}}, \varepsilon / 2\right)$. Moreover, by (2.1), there exists $\eta \in\left(0, \eta_{1}\right)$, such that $\omega_{\lambda}\left(J_{\lambda}\left(B_{0}\right)\right) \subset \bar{N}\left(A_{\widehat{\lambda}}, \widehat{\varepsilon} / 2\right)$, for each $\lambda \in \Lambda$, with $d_{\Lambda}(\widehat{\lambda}, \lambda) \leq \eta$. Then for each $\lambda \in \Lambda$ with $d_{\Lambda}(\widehat{\lambda}, \lambda) \leq \eta, B_{\lambda}$ attracts the compact subset of $M$ and $A_{\lambda}=\omega_{\lambda}\left(B_{\lambda}\right)$ is a global attractor for $U_{\lambda}$. Finally since $B_{\lambda} \subset \bar{N}\left(A_{\widehat{\lambda}}, \varepsilon / 2\right)$, we deduce that

$$
A_{\lambda} \subset \bar{N}\left(A_{\widehat{\lambda}}, \varepsilon / 2\right)
$$

and the result follows. 
Let $\rho: M \rightarrow[0,+\infty)$ be a continuous function. Define

$$
M_{0}:=\{x \in M: \rho(x)>0\} \text { and } \partial M_{0}:=\{x \in M: \rho(x)=0\} .
$$

Let $\{U(t)\}_{t \in I}$ be a continuous semi-flow such that

$$
U(t)\left(M_{0}\right) \subset M_{0}, \quad \forall t \geq 0 .
$$

A subset $B \subset M_{0}$ is said to be $\rho$-strongly bounded if $B$ is bounded in $(M, d)$ and $\inf _{x \in B} \rho(x)>0$.

Definition 2.10 $\{U(t)\}_{t \in I}$ is said to be $\rho$-uniformly persistent if there exists $\varepsilon>0$ such that $\liminf _{t \rightarrow+\infty} \rho(U(t)(x)) \geq \varepsilon, \forall x \in M_{0}$.

The following result on the existence of global attractors in $\left(M_{0}, d\right)$ is proved in ([36], Theorem 3.7).

Theorem 2.11 Assume that $U$ is asymptotically smooth, $\rho$-uniformly persistent, and that $U$ has a global attractor $A$ in $(M, d)$. Then $U$ has a global attractor $A_{0}$ in $\left(M_{0}, d\right)$. Moreover, for each subset $B$ of $M_{0}$, if there exists $t \geq 0$ such that $\gamma^{+}(U(t)(B))$ is $\rho$-strongly bounded, then $A_{0}$ attracts $B$ for $U$.

From now on we assume that Assumption A is satisfied. By Theorem 2.11, it follows that, for each $\lambda \in \Lambda, U_{\lambda}$ has a global attractor $A_{0 \lambda}$ in $\left(M_{0}^{\lambda}, d\right)$, and the first part of Theorem 1.1 follows. So it remains to prove the upper semi-continuity of the family $\left\{A_{0 \lambda}\right\}_{\lambda \in \Lambda}$ at $\widehat{\lambda}$, that is to show that

$$
\delta\left(A_{0 \lambda}, A_{0 \widehat{\lambda}}\right)=\sup _{x \in A_{0 \lambda}} \inf _{y \in A_{0 \widehat{\lambda}}} d(x, y) \rightarrow 0 \text { as } \lambda \rightarrow \widehat{\lambda} .
$$

In practice as for uniform persistence (see [36], Proposition 3.2), we may use the following equivalent conditions.

Lemma 2.12 Let Assumption A be satisfied. Then the following statements are equivalent:

(a) There exist $\varepsilon>0$ and $\eta>0$, such that

$$
\lim _{t \rightarrow+\infty} \inf \rho_{\lambda}\left(U_{\lambda}(t) x\right) \geq \varepsilon, \quad \forall x \in M_{0}^{\lambda}, \forall \lambda \in \bar{B}_{\Lambda}(\widehat{\lambda}, \eta) .
$$

(b) There exist $\varepsilon>0$ and $\eta>0$, such that

$$
\lim _{t \rightarrow+\infty} \sup \rho_{\lambda}\left(U_{\lambda}(t) x\right) \geq \varepsilon, \quad \forall x \in M_{0}^{\lambda}, \forall \lambda \in \bar{B}_{\Lambda}(\widehat{\lambda}, \eta) .
$$

(c) There exist $\varepsilon>0$ and $\eta>0$, such that for each $x \in M_{0}^{\lambda}$ and each $\lambda \in \bar{B}_{\Lambda}(\widehat{\lambda}, \eta)$,

$$
\text { if } 0<\rho_{\lambda}(x)<\varepsilon \text {, there exists } t_{0}=t_{0}(x, \lambda) \geq 0 \text {, such that } \rho_{\lambda}\left(U_{\lambda}\left(t_{0}\right)(x)\right) \geq \varepsilon \text {. }
$$

Proof The implications (a) $\Leftrightarrow$ (c) and (b) $\Rightarrow$ (a) are obvious. We prove that (a) implies (b). Let $\varepsilon>0$ and $\eta>0$ satisfy

$$
\lim _{t \rightarrow+\infty} \sup \rho_{\lambda}\left(U_{\lambda}(t)(x)\right) \geq \varepsilon, \quad \forall x \in M_{0}, \quad \forall \lambda \in B_{\Lambda}(\widehat{\lambda}, \eta) .
$$

Assume that there exist sequences $\left\{x_{m}\right\}_{m \geq 0} \subset M$ and $\left\{\lambda_{m}\right\}_{m \geq 0} \subset B_{\Lambda}(\widehat{\lambda}, \eta)$, such that

$$
\begin{aligned}
& \lambda_{m} \rightarrow \widehat{\lambda} \text { as } m \rightarrow+\infty, \\
& u_{m}:=\lim _{t \rightarrow+\infty} \inf \rho_{\lambda_{m}}\left(U_{\lambda_{m}}(t)\left(x_{m}\right)\right) \rightarrow 0 \text { as } m \rightarrow+\infty .
\end{aligned}
$$


Let $m_{0} \geq 0$ such that for each $m \geq m_{0}, u_{m}<\varepsilon / 2$. Then for each $m \geq m_{0}$, we can find $t_{m} \in I$ and $l_{m} \in I$, such that, if $y_{m}=U_{\lambda_{m}}\left(t_{m}\right)\left(x_{m}\right)$, then

$$
d\left(y_{m}, A_{\lambda_{m}}\right) \leq \frac{1}{m}, \rho_{\lambda_{m}}\left(y_{m}\right) \geq \varepsilon / 2, \quad \rho_{\lambda_{m}}\left(U_{\lambda_{m}}(l) y_{m}\right) \leq \varepsilon / 2, \quad \forall l \in\left(0, l_{m}\right] \cap I,
$$

and

$$
\rho_{\lambda_{m}}\left(U_{\lambda_{m}}\left(l_{m}\right) y_{m}\right) \leq u_{m}+\frac{1}{m+1} .
$$

Since $\left\{A_{\lambda}\right\}_{\lambda \in \Lambda}$ is upper semi-continuous at $\widehat{\lambda}$, there exists a subsequence (that we still denote $\left.y_{m}\right)$, satisfying,

$$
y_{m} \rightarrow y \in A_{\widehat{\lambda}} \text { as } m \rightarrow+\infty .
$$

We set $\widehat{l}:=\liminf _{m \rightarrow+\infty} l_{m}$. If $\widehat{l}<+\infty$, we have

$$
\rho_{\widehat{\lambda}}(y) \geq \varepsilon / 2 \text { and } \rho_{\widehat{\lambda}}\left(U_{\widehat{\lambda}}(\widehat{l}) y\right)=0,
$$

which is impossible because $y \in M_{0}^{\widehat{\lambda}}$ and $M_{0}^{\widehat{\lambda}}$ is positively invariant by $U_{\widehat{\lambda}}$. If $\widehat{l}=+\infty$, we have

$$
y \in M_{0}^{\widehat{\lambda}} \text { and } \lim _{n \rightarrow+\infty} \sup \rho_{\widehat{\lambda}}\left(U_{\widehat{\lambda}}(t)(y)\right) \leq \varepsilon / 2, \quad \forall t>0,
$$

which contradicts (2.2).

Proof of Theorem 1.1 Assume that $\left\{A_{0 \lambda}\right\}_{\lambda \in \Lambda}$ is upper semi-continuity at $\widehat{\lambda}$. Let $\varepsilon$ $\in\left(0, \inf _{x \in A_{0 \widehat{\lambda}}} \rho_{\lambda}(x)\right)$. Assume by contradiction that we can find a sequence $\lambda_{n} \rightarrow \widehat{\lambda}$ and $x_{n} \in M_{0}^{\lambda_{n}}$ such that

$$
\lim _{t \rightarrow+\infty} \inf \rho_{\lambda_{n}}\left(U_{\lambda_{n}}(t) x_{n}\right) \leq \varepsilon
$$

So, for each $n \geq 0$, the omega-limit set $\omega\left(x_{n}\right)$ for $U_{\lambda}$ contains a point $y_{n} \in A_{0 \lambda_{n}}$ with $\rho_{\lambda_{n}}\left(y_{n}\right) \leq \varepsilon$. But since $\rho$ is continuous, by using the upper semi-continuity of $\left\{A_{0 \lambda}\right\}_{\lambda \in \Lambda}$ at $\widehat{\lambda}$, we can find $y \in A_{0 \widehat{\lambda}} \subset M_{0}^{\widehat{\lambda}}$ with $\rho_{\lambda_{n}}(y) \leq \varepsilon$, which leads to a contradiction.

Conversely assume that there exists $\varepsilon>0$ such that

$$
\lim _{t \rightarrow+\infty} \inf \rho_{\lambda}\left(U_{\lambda}(t)(x)\right) \geq \varepsilon, \quad \forall x \in M_{0}^{\lambda}, \forall \lambda \in B_{\Lambda}(\widehat{\lambda}, \eta) .
$$

We first prove that there exist $\widehat{\varepsilon} \in(0, \varepsilon)$ and $\widehat{\eta} \in(0, \eta)$, such that

$$
\rho_{\widehat{\lambda}}(x) \geq \widehat{\varepsilon}, \quad \forall x \in \bigcup_{\lambda \in B_{\Lambda}(\widehat{\lambda}, \widehat{\eta})} A_{0 \lambda} .
$$

Assume, by contradiction, that this is not true. Then we can find two sequences $\lambda_{n} \rightarrow \widehat{\lambda}$ and $x_{n} \in A_{0 \lambda_{n}}$, such that

$$
u_{n}:=\rho_{\widehat{\lambda}}\left(x_{n}\right) \rightarrow 0 \text { as } n \rightarrow+\infty .
$$

Since $\left\{A_{\lambda}\right\}_{\lambda \in \Lambda}$ is upper semi-continuous at $\widehat{\lambda}$, there exists subsequence (that we still denote $x_{n}$ ) such that,

$$
x_{n} \rightarrow x \in A_{\widehat{\lambda}},
$$


Since $\rho$ is continuous, we deduce that

$$
\rho_{\lambda_{n}}\left(x_{n}\right) \rightarrow 0 \text { as } n \rightarrow+\infty .
$$

So for all $n \geq 0$ large enough, we have

$$
\rho_{\lambda_{n}}\left(x_{n}\right)<\varepsilon / 2, \quad \forall n \geq 0 .
$$

Since $A_{0 \lambda_{n}}$ is invariant by $U_{\lambda_{n}}$, for each $n \geq 0$, we can find $y_{n} \in A_{0 \lambda_{n}}$ and $l_{n} \in I$, such that

$$
x_{n}=U_{\lambda_{n}}\left(l_{n}\right) y_{n}, \rho_{\lambda_{n}}\left(y_{n}\right) \geq \varepsilon / 2, \quad \text { and } \rho_{\lambda_{n}}\left(U_{\lambda_{n}}(l)\left(y_{n}\right)\right) \leq \varepsilon / 2, \forall l \in\left(0, l_{n}\right],
$$

Otherwise we can find a negative orbit $\left\{u^{n}(-t)\right\}_{t \geq 0} \subset A_{0 \lambda_{n}}$ such that

$$
\begin{aligned}
& x_{n}=u^{n}(0), \text { and } u^{n}(l-t)=U_{\lambda_{n}}(l)\left(u^{n}(-t)\right), \quad \forall t, l \geq 0, \text { with } l-t \leq 0, \\
& \text { and } \rho_{\lambda_{n}}\left(u^{n}(-t)\right)<\varepsilon / 2, \quad \forall t \geq 0 .
\end{aligned}
$$

We deduce that, for each point $z \in \alpha\left(x^{n}\right)=\bigcap_{t \geq 0} \overline{\bigcup_{s \geq t}\left\{u^{n}(-s)\right\}} \subset A_{0 \lambda_{n}}$, we have

$$
\rho_{\lambda_{n}}\left(U_{\lambda_{n}}(t)(z)\right) \leq \varepsilon / 2, \quad \forall t \geq 0,
$$

which contradicts (2.3). Now since $\left\{A_{\lambda}\right\}_{\lambda \in \Lambda}$ is upper semi-continuous at $\widehat{\lambda}$, there exists a subsequence (that we still denote $y_{n}$ ) such that,

$$
y_{n} \rightarrow y \in A_{\widehat{\lambda}} \text { as } n \rightarrow+\infty .
$$

We denote $\widehat{l}=\lim _{n \rightarrow+\infty} \inf l_{n}$. Then if $\widehat{l}<+\infty$, we have

$$
\rho_{\widehat{\lambda}}(y) \geq \varepsilon / 2 \text { and } \rho_{\widehat{\lambda}}\left(U_{\widehat{\lambda}}(\widehat{l})(y)\right)=0,
$$

which is impossible, since $y \in M_{0}^{\widehat{\lambda}}$ and $M_{0}^{\widehat{\lambda}}$ is positively invariant by $U_{\widehat{\lambda}}$. If $\widehat{l}=+\infty$, we have

$$
y \in M_{0}^{\widehat{\lambda}} \text { and } \lim _{t \rightarrow+\infty} \sup \rho_{\widehat{\lambda}}\left(U_{\widehat{\lambda}}(t)(y)\right) \leq \varepsilon / 2,
$$

which contradicts (2.3). Finally, there exist $\widehat{\varepsilon} \in(0, \varepsilon)$ and $\widehat{\eta} \in(0, \eta)$, such that

$$
\rho_{\widehat{\lambda}}(x) \geq \widehat{\varepsilon}, \quad \forall x \in \cup_{\lambda \in B_{\Lambda}(\widehat{\lambda}, \widehat{\eta})} A_{0 \lambda} .
$$

We set $V:=\left\{x \in M: \rho_{\widehat{\lambda}}(x) \geq \widehat{\varepsilon}\right\}$. Then $V$ is closed in $(M, d)$, the subset $C:=V \cap A_{\widehat{\lambda}}$ is a compact subset of $M_{0 \widehat{\lambda}}$, and

$$
\delta\left(A_{0 \lambda}, C\right) \rightarrow 0 \text { as } \lambda \rightarrow \widehat{\lambda} .
$$

Thus, Proposition 2.7 implies the result.

\section{Perturbation of a Globally Stable Steady State}

In this section, motivated by the results in [42], we study the perturbation of a globally stable steady state.

Let $(X,\|\|$.$) be a Banach space. For any subsets A \subset B \subset X$ and any map $g: B \rightarrow X$, we set

$$
\|g\|_{\text {Lip }, A}=\sup _{x, y \in A: x \neq y} \frac{\|g(x)-g(y)\|}{\|x-y\|} .
$$


Proof of Theorem 1.2 We recall that $r(L)=\lim _{n \rightarrow+\infty}\left\|L^{n}\right\|_{\mathcal{L}(X)}^{1 / n}$. Let $c \in(r(L), 1)$. We consider the norm

$$
|x|=\sup _{n \geq 0} c^{-n}\left\|L^{n} x\right\|, \quad \forall x \in X .
$$

Then $|$.$| is a norm equivalent to \|$.$\| , and |L x| \leq c|x|, \forall x \in X$. So without loss of generality, we can assume that $\|L\|_{\mathcal{L}(X)}<1$. Let $k \in\left(0,1-\|L\|_{\mathcal{L}(X)}\right)$, and let $r>0$ be fixed such that

$$
\left.\left\|U_{\lambda}\left(t_{0}\right)-L\right\|_{L i p, \bar{B}_{M}\left(\bar{x}_{\widehat{\lambda}}, r\right)} \leq k, \quad \forall \lambda \in \bar{B}_{\Lambda} \widehat{(\lambda}, r\right) .
$$

Then for each $\lambda \in \bar{B}_{\Lambda}(\widehat{\lambda}, r)$ and each $x, y \in \bar{B}_{M}\left(\bar{x}_{\widehat{\lambda}}, r\right)$, we have

$$
\left\|U_{\lambda}\left(t_{0}\right) x-U_{\lambda}\left(t_{0}\right) y\right\| \leq \alpha\|x-y\|,
$$

with $\alpha=k+\|L\|_{\mathcal{L}(X)}<1$.

Since the family $\left\{A_{0 \lambda}\right\}_{\lambda \in \Lambda}$ is upper semi-continuous, we can find $\eta \in(0, r)$, such that

$$
A_{0 \lambda} \subset \bar{B}_{M}\left(\bar{x}_{\widehat{\lambda}}, r / 2\right), \quad \forall \lambda \in \bar{B}_{\Lambda}(\widehat{\lambda}, \eta) .
$$

Since $A_{0 \lambda}$ is invariant by $U_{\lambda}$, we obtain for each $\lambda \in \bar{B}_{\Lambda}(\widehat{\lambda}, \eta)$ that

$$
\begin{aligned}
\operatorname{diam}\left(A_{0 \lambda}\right) & :=\sup _{x, y \in A_{\lambda}}\|x-y\|=\sup _{x, y \in A_{0 \lambda}}\left\|U_{\lambda}\left(t_{0}\right) x-U_{\lambda}\left(t_{0}\right) y\right\| \\
& \leq \alpha \operatorname{diam}\left(A_{0 \lambda}\right) .
\end{aligned}
$$

We deduce that for each $\lambda \in \bar{B}_{\Lambda} \widehat{(\lambda, \eta)}, \operatorname{diam}\left(A_{0 \lambda}\right)=0$, so there exists $\bar{x}_{\lambda} \in M$, such that $A_{0 \lambda}=\left\{\bar{x}_{\lambda}\right\}$. Since $A_{0 \lambda}$ is invariant by $U_{\lambda}$, it follows that $\bar{x}_{\lambda}$ is an equilibrium. Moreover $\bar{x}_{\lambda} \in \bar{B}_{M}\left(\bar{x}_{\hat{\lambda}}, r / 2\right)$, and $U_{\lambda}$ is a continuous semi-flow, so by using (3.1), it follows that $\bar{x}_{\lambda}$ is stable.

We now turn to the case where 1 is a simple dominant eigenvalue of $L$. We first recall some reduction results, which hold under the following assumption.

Assumption C Let $L$ be a bounded linear operator on a Banach space $X$. We assume that $X$ can be written as $X=X_{1} \oplus X_{2}$, where $X_{1}$ and $X_{2}$ are closed subspaces of $X$, which are positively invariant by $L$, and

$$
a=\sup _{\lambda \in \sigma\left(L_{1}\right)}|\lambda|<\inf _{\lambda \in \sigma\left(L_{2}\right)}|\lambda|=b \leq 1,
$$

where $L_{i}=\left.L\right|_{X_{i}} \in L\left(X_{i}\right)$, for $i=1,2$.

We denote by $P \in \mathcal{L}\left(X_{i}\right)$ the bounded linear projector, such that

$$
P(X)=X_{2}, \quad \text { and } \quad(I-P)(X)=X_{1} .
$$

Lemma 3.1 ([28], Theorem 3.3) Let Assumption C be satisfied. Let $M$ be a closed bounded set of $X$. Let $\eta \in(a, b)$ be fixed. Then there exists a constant $C_{1}=C_{1}(L, \eta)>0$, such that for each map $T: M \rightarrow M$ satisfying

$$
C_{1}\|T-L\|_{L i p, M}<1 \text { and } T(M)=M,
$$

then $T$ is bijective, and there exists a Lipschitz continuous map $\phi: P(M) \rightarrow(I d-P)(M)$ such that

$$
M=\{y+\phi(y): y \in P(M)\} .
$$


For each $\eta>0$, we define the Banach space $Y_{\eta}^{-}(X)$ of all sequences $\left\{y_{-p}\right\}_{p \in \mathbb{N}}$ such that

$$
\|y\|_{Y_{\eta}^{-}(X)}=\sup _{p \geq 0} \eta^{p}\left\|y_{-p}\right\|<+\infty .
$$

For each $\eta>0$ and each map $T: X \rightarrow X$, we denote

$$
M_{\eta}(T)=\left\{y_{0}: \text { there is a } T \text {-negative orbit } y \in Y_{\eta}^{-}(X) \text { passing through } y_{0}\right\} .
$$

Lemma 3.2 ([50], Theorems 5 and 6) Let Assumption $C$ be satisfied. Let $\eta \in(a, b)$ be fixed. Then there exists $C_{1}=C_{1}(A, \eta)>0$, such that for each map $T: X \rightarrow X$ satisfying

$$
T-L \text { is bounded and } C_{1}\|T-L\|_{L i p, X}<1 \text {, }
$$

then $T$ is a bijection on $M_{\eta}$, and there exists a map $\phi_{\eta} \in \operatorname{Lip}\left(X_{2}, X_{1}\right)$ such that for all $x \in M_{\eta}$,

$$
M_{\eta}(T)=\left\{x+\phi_{\eta}(x): x \in X_{2}\right\} .
$$

Moreover, there exists a continuous map $H: X \rightarrow M_{\eta}$ such that for each $x \in X$,

$$
M_{\eta} \cap \widetilde{M}_{1 / \eta}(x)=\{H(x)\},
$$

where

$$
\tilde{M}_{1 / \eta}(x)=\left\{y \in X: \sup _{n \geq 0} \eta^{-n}\left\|T^{n}(y)-T^{n}(x)\right\|<+\infty\right\} .
$$

Lemma 3.3 Let $J$ be a closed interval in $\mathbb{R}$. Let $I=\mathbb{R}$ or $I=\omega \mathbb{N}$ for some $\omega>0$. Let $\{V(t)\}_{t \in I}$ be a strongly monotone continuous semi-flow on $J$ (i.e. $x<y \Rightarrow V(t) x<$ $V(t) y, \forall t \in I)$. Let $A$ be a compact subset of $J$. Set $a=\inf A$ and $b=\sup A$. Then we have the following:

(i) If $A$ is invariant for $V$, then $a$ and $b$ are equilibria of $V$. Moreover, if there exist $x \in[a, b]$ and $\tau \in I \backslash\{0\}$, such that $V(\tau) x>x(V(\tau) x<x)$ then there exist two equilibria of $V$, $c, d \in[a, b]$ with $c<x<d$ such that $V(t) y>y(V(t) y<y), \forall t \in I \backslash\{0\}, \forall y \in(c, d)$.

(ii) If $A$ is invariant and internally chain transitive for $V$, then

$$
A=[a, b] \text {, and } V(t) x=x, \quad \forall t \in I \backslash\{0\}, \forall x \in[a, b] .
$$

Proof The proof for (i) is immediate. To prove (ii), assume that there exist $\tau>0$ and $x \in(a, b)$, such that $V(\tau) x \neq x$. If $V(\tau) x>x$ then $b$ is not chained to $a$, and if $V(\tau) x<x$ then $a$ is not chained to $b$. So $V(t) x=x, \forall t \in I, \forall x \in[a, b]$. By using again the fact that $b$ is chained to $a$, we deduce that $A$ is dense in $[a, b]$, and since $A$ is closed, we deduce that $A=[a, b]$.

Proof of Theorem 1.4 We set $T_{\lambda}(x)=U_{\lambda}\left(t_{0}\right)(x), \forall(\lambda, x) \in \Lambda \times M$. By using Assumption (C) of Theorem 1.4, we deduce that there exists $\widehat{\delta}>0$, such that, if $\lambda \in \bar{B}_{\Lambda}(\widehat{\lambda}, \widehat{\delta})$, and $\widehat{M} \subset \bar{B}_{M}\left(\bar{x}_{\widehat{\lambda}}, \widehat{\delta}\right)$ is invariant for $T_{\lambda}$, then we can apply Lemma 3.1. But since $A_{\widehat{\lambda}}=\left\{\bar{x}_{\widehat{\lambda}}\right\}$ and the family $\left\{A_{0 \lambda}\right\}_{\lambda \in \Lambda}$ is upper semi-continuous at $\widehat{\lambda}$, we deduce that there exists $\delta \in(0, \widehat{\delta})$, such that

$$
A_{0 \lambda} \subset \bar{B}_{M}\left(\bar{x}_{\widehat{\lambda}}, \widehat{\delta}\right), \quad \forall \lambda \in \bar{B}_{\Lambda}(\widehat{\lambda}, \delta) .
$$

We can now apply Lemma3.1 to $T_{\lambda}$ with $M=A_{0 \lambda}$ and $\lambda \in \bar{B}_{\Lambda}(\widehat{\lambda}, \delta)$. We deduce that $\left.\forall \lambda \in \bar{B}_{\Lambda} \widehat{(\lambda}, \delta\right)$, there exists $\phi_{\lambda} \in \operatorname{Lip}\left(P\left(A_{0 \lambda}\right),(I d-P)\left(A_{0 \lambda}\right)\right)$ such that

$$
A_{0 \lambda}=\left\{y+\phi_{\lambda}(y): y \in P\left(A_{0 \lambda}\right)\right\} \text {. }
$$


Moreover, $T_{\lambda}=U_{\lambda}\left(t_{0}\right)$ is a bijection from $A_{0 \lambda}$ into itself. We claim that for each $t \in I$, $U_{\lambda}(t)$ is a bijection from $A_{0 \lambda}$ into itself. Let $t \in\left[0, t_{0}\right) \cap I$ be fixed. Since $U_{\lambda}(t) A_{0 \lambda}=A_{0 \lambda}$, it is sufficient to prove that $U_{\lambda}(t)$ is one to one. Let $x, y \in A_{0 \lambda}$ be fixed. Assume that $U_{\lambda}(t) x=U_{\lambda}(t) y$, then $U_{\lambda}\left(t_{0}\right) x=U_{\lambda}\left(t_{0}-t\right) U_{\lambda}(t) x=U_{\lambda}\left(t_{0}-t\right) U_{\lambda}(t) y=U_{\lambda}\left(t_{0}\right) y$, and since $U_{\lambda}\left(t_{0}\right)$ is one to one, we deduce that $x=y$. So $U_{\lambda}(t)$ is one to one.

Let $v \in X, v^{*} \in X^{*}$, with $v^{*}(v)=1$, such that $P(x)=v^{*}(x) v, \forall x \in X$. We set

$$
J_{\lambda}=\left\{v^{*}(x): x \in A_{0 \lambda}\right\}
$$

and $\left\{V_{\lambda}(t)\right\}_{t \geq 0}$ a family of maps on $J_{\lambda}$, defined by

$$
V_{\lambda}(t)(\alpha)=v^{*}\left(U_{\lambda}(t)\left(\alpha v+\phi_{\lambda}(\alpha v)\right)\right), \quad \forall \alpha \in J_{\lambda} .
$$

For each $\lambda \in \bar{B}_{\Lambda}(\widehat{\lambda}, \delta),\left\{V_{\lambda}(t)\right\}_{t \in I}$ is a continuous semiflow on $J_{\lambda}$, and since $A_{0 \lambda}$ is compact connected, $J_{\lambda}$ is a compact interval in $\mathbb{R}$, which is invariant by $V_{\lambda}$.

Let $t \in I$ be fixed. Since $U_{\lambda}(t)$ is a bijection from $A_{0 \lambda}$ into itself, $V_{\lambda}(t)$ is also a bijection from $J_{\lambda}$ into itself. So $V_{\lambda}(t)$ is either increasing or decreasing. If the Assumption D-(1) of Theorem 1.4 is satisfied, then $v^{*}\left(\bar{x}_{\lambda}\right)$ is a fixed point of $V_{\lambda}(t)$, which is extreme in $J_{\lambda}$, and $V_{\lambda}(t)\left(J_{\lambda}\right) \subset J_{\lambda}$, so $V_{\lambda}(t)$ is increasing. If the Assumption D-(2) of Theorem 1.4 is satisfied, then $J_{\lambda i} \equiv\left\{v^{*}(x): x \in E_{i \lambda}\right\}$, for $i=1,2$, is a compact interval, $V_{\lambda}(t)\left(J_{\lambda i}\right) \subset J_{\lambda i}$, and $J_{\lambda 1} \cap J_{\lambda 2}=\emptyset$. So $V_{\lambda}(t)$ has two distinct equilibria, and $V_{\lambda}(t)$ is increasing. Assertions (i) and (ii) of Theorem 1.4 now follow from Lemma 3.3-(i) applied to $\left\{V_{\lambda}(t)\right\}_{t \in I}$.

We now prove assertion (iii) of Theorem 1.4. Assume that $A_{0 \lambda}$ contains at most a finite number of equilibria. Let $x \in M$ be such that $d\left(U_{\lambda}(t)(x), A_{0 \lambda}\right) \rightarrow 0$ as $t \rightarrow+\infty$. By Lemma 2.1, we know that the omega-limit set $\omega(x)$ of $\left\{U_{\lambda}(t)(x)\right\}_{t \in I}$ is compact and internally chain transitive for $U_{\lambda}$, and $\omega(x) \subset A_{0 \lambda}$. We set

$$
J_{\omega(x)}=\left\{v^{*}(x): x \in \omega(x)\right\} \subset J_{\lambda} .
$$

Then $J_{\omega(x)}$ is compact, and internally chain transitive for $V_{\lambda}$. By using the fact that $V_{\lambda}$ is increasing, and by Lemma 3.3-(ii), we deduce that there exist $a, b \in J_{\lambda}, a \leq b$, such that

$$
J_{\omega(x)}=[a, b] \text { with } V_{\lambda}(t)(x)=x, \quad \forall x \in[a, b], \forall t \in I .
$$

But since $U_{\lambda}$ has a finite number of equilibria in $A_{0 \lambda}$, we deduce that $V_{\lambda}$ has a finite number of equilibria, so $a=b$.

Assertion (iv) of Theorem 1.4 follows from Lemma 3.3-(i). So, it remains to prove assertion (v). We proceed by extension and truncation and we apply Lemma 3.2. Since $V$ is a neighborhood of $\left(\widehat{\lambda}, \bar{x}_{\widehat{\lambda}}\right)$ in $\Lambda \times X$, there exists $\delta^{*}>0$, such that $\left.\bar{B}_{X}\left(\bar{x}_{\widehat{\lambda}}, \delta^{*}\right) \times \bar{B}_{\Lambda} \widehat{(\lambda,}, \delta^{*}\right) \subset V$. Let $\chi:[0,+\infty) \rightarrow[0,+\infty)$ be a Lipschitz continuous function, such that

$$
\begin{aligned}
& \chi(s)=0, \quad \text { if } s \geq 2, \\
& \chi(s) \in[0,1], \quad \text { if } s \in[1,2], \\
& \chi(s)=1, \quad \text { if } 0 \leq s \leq 1
\end{aligned}
$$

We set for each $R>0$ and each $\lambda \in B_{\Lambda}\left(\widehat{\lambda}, \delta^{*}\right)$

$$
\widetilde{T}_{R, \lambda}(x)= \begin{cases}L x+\chi\left(R^{-1}\left\|x-\bar{x}_{\widehat{\lambda}}\right\|\right)\left(\widehat{T}_{\lambda}(x)-L x\right), & \text { if } x \in B_{X}\left(\bar{x}_{\widehat{\lambda}}, \delta^{*}\right) \\ 0, & \text { otherwise. }\end{cases}
$$


By using the same arguments as in the proof of [30,Proposition 4.4], we obtain

(1) $\widetilde{T}_{R, \lambda}(x)=T_{\lambda}(x), \forall x \in \bar{B}_{M}\left(\bar{x}_{\widehat{\lambda}}, R\right), \forall R>0$.

(2) There exists $R_{0}>0$, such that $\widetilde{T}_{R, \lambda}-L$ is

Lipschitz and bounded, $\forall \lambda \in \bar{B}_{\Lambda}\left(\widehat{\lambda}, \delta^{*}\right), \forall R \in\left(0, R_{0}\right)$.

(3) $\lim _{R \rightarrow 0^{+}} \sup _{\lambda \in \Lambda: d_{\Lambda}(\widehat{\lambda}, \lambda) \leq R}\left\|\widetilde{T}_{R, \lambda}-L\right\|_{\text {Lip }, X}=0$.

Let $\eta \in(r(L(I-P)), 1)$ be fixed. Let $R>0$ be fixed, such that for each $\lambda \in \Lambda: d_{\Lambda}(\widehat{\lambda}, \lambda) \leq$ $R$, Lemma 3.2 applies to $\widetilde{T}_{R, \lambda}$. Then there exists a map $\phi_{\lambda} \in \operatorname{Lip}(P(X),(I d-P)(X))$ such that

$$
M_{\eta, \lambda}=\left\{x+\phi_{\lambda}(x): x \in P(X)\right\} .
$$

Moreover, $\widetilde{T}_{R, \lambda}$ is a bijection from $M_{\eta}$ into itself. We define the continuous map $h_{\lambda}: \mathbb{R} \rightarrow \mathbb{R}$ by

$$
h_{\lambda, R}(\alpha)=v^{*}\left(\widetilde{T}_{R, \lambda}\left(\alpha v+\phi_{\lambda}(\alpha v)\right)\right), \quad \forall \alpha \in \mathbb{R} .
$$

We deduce that $h_{\lambda, R}$ is continuous and invertible, so $h_{\lambda, R}$ is either strictly increasing, or strictly decreasing. Now by using the upper semi-continuity of the family $\left\{A_{0 \lambda}\right\}_{\lambda \in \Lambda}$ at $\widehat{\lambda}$, we deduce that there exists $\delta \in(0, R)$, such that for all $\lambda \in \bar{B}_{\Lambda}(\widehat{\lambda}, \delta)$,

$$
A_{0 \lambda} \subset B_{M}\left(\bar{x}_{\hat{\lambda}}, R / 2\right) .
$$

In particular by the definition of the center manifold $M_{\eta}$, and since $T_{\lambda}\left(A_{0 \lambda}\right)=A_{0 \lambda}$ and $A_{0 \lambda}$ is bounded, we deduce that $A_{0 \lambda} \subset M_{\eta}$. Let $x \in M$ be such that $d\left(T_{\lambda}^{n}(x), A_{0 \lambda}\right) \rightarrow 0$ as $n \rightarrow+\infty$. Then there exists $m \geq 0$, such that

$$
T_{\lambda}^{k+m}(x) \in B_{M}\left(\bar{x}_{\widehat{\lambda}}, R\right), \quad \forall k \geq 0 .
$$

So in particular if we set $y=T_{\lambda}^{m}(x)$, we have

$$
T_{\lambda}^{k}(y)=\widetilde{T}_{R, \lambda}^{k}(y), \quad \forall k \geq 0 .
$$

If $A_{0 \lambda}$ is a single point (which is possible under Assumption D-(1) of Theorem 1.4), then $T_{\lambda}^{n}(x) \rightarrow \bar{x}$ as $n \rightarrow+\infty$, and there is nothing to prove.

Otherwise, if $A_{0 \lambda}$ is not reduced to a single point, we know from the first part of the proof that $h_{\lambda, R}$ restricted to $J_{\lambda}=\left\{v^{*}(x): x \in A_{0 \lambda}\right\}$ is increasing. We deduce that $h_{\lambda, R}$ is increasing on $\mathbb{R}$. So for all $z \in M_{\eta}$, if $\left\{\widetilde{T}_{R, \lambda}^{n}(z)\right\}_{n \geq 0}$ is bounded, then this sequence converges to some fixed point of $\widetilde{T}_{R, \lambda}$. By Lemma3.2, we know that there exist $z \in M_{\eta}$ and $C>0$ such that

$$
\left\|\widetilde{T}_{R, \lambda}^{n}(y)-\widetilde{T}_{R, \lambda}^{n}(z)\right\|<C \eta^{n} \rightarrow \text { 0as } n \rightarrow+\infty .
$$

We deduce that $\left\{\widetilde{T}_{R, \lambda}^{n}(z)\right\}_{n \geq 0}$ is a bounded sequence, so this sequence converges to some fixed point $\bar{x} \in M_{\eta}$ of $\widetilde{T}_{R, \lambda}$. So

$$
T_{\lambda}^{n}(y)=\widetilde{T}_{R, \lambda}^{n}(y) \rightarrow \bar{x} \text { as } n \rightarrow+\infty .
$$

Finally, since $d\left(T_{\lambda}^{n}(x), A_{0 \lambda}\right) \rightarrow 0$ as $n \rightarrow+\infty$, we deduce that $\bar{x} \in A_{0 \lambda}$, so $\bar{x}$ is also a fixed point of $T_{\lambda}$. Now by using Lemma3.3-(i), we deduce that $\bar{x}$ is an equilibrium of $U_{\lambda}$, and the result follows. 


\section{Applications}

We now present two examples of applications to age-structured population dynamic models. We refer to $[23,52]$ for studies on age-structured models by using Volterra integral equations. We also refer to [31-33,45] and to the references therein for the integrated semi-group approach.

From now on, for any interval $I \subset \mathbb{R}$, we denote by $C_{B U}(I, \mathbb{R})$ the set of bounded and uniformly continuous maps from $I$ into $\mathbb{R}$.

Example 1 We consider the age-structured model introduced by Liu and Cohen [27],

$$
\left\{\begin{array}{l}
\frac{\partial u}{\partial t}+\frac{\partial u}{\partial a}=-\left(\mu(a)+\int_{0}^{c} \gamma(a, s) u(t, s) d s\right) u(t, a), a \in(0, c), \\
u(t, 0)=\int_{0}^{c} \beta(a) \exp \left(-\int_{0}^{c} \widehat{\gamma}(a, s) u(t, s) d s\right) u(t, a) d a, \\
u(0)=\varphi \in L_{+}^{1}(0, c) .
\end{array}\right.
$$

where $c \in(0,+\infty)$ is the maximum attainable age, $\mu(a)$ is the natural mortality rate, $\beta(a)$ is the fertility rate, $\gamma$ and $\widehat{\gamma}$ are saturation terms for the mortality and the fertility respectively. We set

$$
R_{0}(\beta):=\int_{0}^{c} \beta(a) \exp \left(-\int_{0}^{a} \mu(s) d s\right) d a,
$$

and we make the following assumption.

Assumption D We assume that

(a) $\beta_{0} \in C_{B U}([0, c), \mathbb{R}), \beta_{0} \geq 0, R_{0}\left(\beta_{0}\right)=1$, and for each $a \in(0, c)$, there exists $a_{1} \in[a, c)$, such that $\beta_{0}\left(a_{1}\right)>0$.

(b) $\mu \in L_{l o c}^{1}([0, c), \mathbb{R}), \mu \geq 0$, and

$$
\lim _{a \rightarrow c^{-}} \int_{0}^{a} \mu(s) d s=+\infty .
$$

(c) $\gamma, \widehat{\gamma} \in C^{1}\left([0, c]^{2}, \mathbb{R}\right)$, there exists a constant $\delta>0$, such that

$$
0<\delta \beta_{0}(s) \leq \gamma(a, s), \quad \forall a, s \in[0, c] .
$$

Here we consider $\beta$ as a parameter of the system, and we set

$$
\Lambda=\left\{\beta \in C_{B U}([0, c), \mathbb{R}): \beta_{0} \leq \beta \leq 2 \beta_{0}\right\}
$$

Then, as a direct application of Theorem 1.4 and of the results in [34] about the asymptotic smoothness of the semi-flow, we obtain the following result.

Theorem 4.1 Let Assumption $D$ be satisfied. Then there exists $\varepsilon>0$, such that, for each $\beta \in \Lambda$, with $\left\|\beta_{0}-\beta\right\|_{\infty} \leq \varepsilon$ and $R_{0}(\beta)>1$, we have:

(a) The system (4.1) has a unique equilibrium $\bar{u}_{\beta}$ in $L_{+}^{1}(0, c) \backslash\{0\}$.

(b) $\bar{u}_{\beta}$ is globally asymptotically stable for the semi-flow generated by (4.1) in $L_{+}^{1}$ $(0, c) \backslash\{0\}$. 
Sketch of Proof We fix $\rho_{\beta}(\varphi)=1, \forall \varphi \in L_{+}^{1}(0, c)$, and $M=M_{0}=L_{+}^{1}(0, c)$. To prove Assumptions $\mathrm{A}$ and $\mathrm{B}$, one may start by proving that for $\beta=\beta_{0}, 0$ is globally asymptotically stable for the system. By using the results in [34] one may prove that the semi-flow is asymptotically smooth. Finally by applying Proposition 2.9 to the system, we deduce that there exists $\eta>0$, and a family of global attractors $\left\{A_{\beta}\right\}_{\beta \in B_{\Lambda}\left(\beta_{0}, \eta\right)}$ which is upper semicontinuous at $\beta_{0}$. Now since $L_{+}^{1}(0, c)$ is closed and convex, so by using the results in [9] or in [11] we deduce that $A_{\beta}$ is connected. So Assumption(A) of Theorem 1.4 is satisfied. The linearized equation of system (4.1) at 0 is

$$
\left\{\begin{array}{l}
\frac{\partial v}{\partial t}+\frac{\partial v}{\partial a}=-\mu(a) v(t, a), a \in(0, c), \\
v(t, 0)=\int_{0}^{c} \beta(a) v(t, a) d a \\
v(0)=\varphi \in L^{1}(0, c) .
\end{array}\right.
$$

Under Assumptions D-(a) and (b), it is well-known that this system generates an irreducible linear $C^{0}$-semi-group on $L^{1}(0, c)$. It follows that Assumption (C) of Theorem 1.4 is satisfied, where $P$ a positive operator. In particular $0 \in A_{\beta}$, for each $\beta \in B_{\Lambda}\left(\beta_{0}, \eta\right)$, and Assumption D-(1) of Theorem 1.4 holds.

Now when $\beta \in \Lambda$, with $R_{0}(\beta)>1$, one may prove (by studying the linearized equation of System (4.1)) that there is uniform persistence in $L_{+}^{1}(0, c) \backslash\{0\}$. It follows that this system has a global attractor $A_{0 \beta}$ in $L_{+}^{1}(0, c) \backslash\{0\}$. Moreover (see [28]), there exists $\eta_{1}>0$ such that, for each $\beta \in \Lambda$, satisfying $\left\|\beta_{0}-\beta\right\|_{\infty} \leq \eta_{1}$ and $R_{0}(\beta)>1$, the system (4.1) has a unique equilibrium $\bar{u}_{\beta}$ in $L_{+}^{1}(0, c) \backslash\{0\}$. It follows that $A_{0 \beta}$ contains a unique equilibrium so we can apply Assertion (iv) of Theorem 1.4 with $B_{\beta}=A_{0 \beta}$. It follows that $A_{0 \beta}=\left\{\bar{u}_{\beta}\right\}$, for $\left\|\beta_{0}-\beta\right\|_{\infty}$ small enough.

Example 2 We now consider the case of an infection-age epidemic model

$$
\left\{\begin{array}{l}
\frac{d S(t)}{d t}=\lambda-\mu S(t)-S(t) \int_{0}^{+\infty} F(a) i(t, a) d a \\
\frac{\partial i}{\partial t}+\frac{\partial i}{\partial a}=-(\mu+v(a)) i(t, a), a \in(0,+\infty), \\
i(t, 0)=S(t) \int_{0}^{+\infty} F(a) i(t, a) d a \\
S(0)=S_{0} \geq 0, i(0)=i_{0} \in L_{+}^{1}(0,+\infty) .
\end{array}\right.
$$

where $S(t)$ is the number of susceptible individuals at time $t, i(t)(a)$ is the density of infected individuals at time $t$ structured with respect to the infection-age $a, \lambda>0$ is the rate of incoming susceptible, $\mu>0$ is the mortality (or exit) rate, $v(a) \in L_{+}^{\infty}(0,+\infty)$ is the mortality (or exit) rate due to the disease, $F(a)$ is the infection rate. We set

$$
R_{0}(F):=\frac{\lambda}{\mu} \int_{0}^{+\infty} F(a) e^{-\int_{0}^{a}(\mu+v(r)) d r} d a,
$$

and make the following assumption.

Assumption $\mathbf{E} \lambda>0, \mu>0, v \in L_{+}^{\infty}(0,+\infty), F_{0} \in C_{B U}([0,+\infty), \mathbb{R}), F_{0} \geq 0$, $R_{0}\left(F_{0}\right)=1$, and, for each $a \in(0,+\infty)$, there exists $a_{1} \in[a,+\infty), F_{0}\left(a_{1}\right)>0$.

This model has been studied by Thieme and Castillo-Chavez [48,49]. More precisely, the local stability of equilibria, the global stability of the disease free equilibrium, and the 
persistence of the disease are investigated in [49]. The global asymptotic stability of the endemic equilibrium has also been proved in [7] under the assumptions that $F$ is uniformly continuous and bounded, and that the map $a \rightarrow F(a) e^{\int_{0}^{a} \mu-v(r) d r}$ is non-decreasing. Here we relax this assumption locally around the bifurcation point.

In order to obtain a global stability result for the endemic equilibrium point, one may directly apply Theorem 1.4 to System (4.2). As before, we consider $F$ as a parameter of the system, and we set

$$
\Lambda=\left\{F \in C_{B U}([0,+\infty), \mathbb{R}): F_{0} \leq F \leq 2 F_{0}\right\} .
$$

If $R_{0}(F) \leq 1$, one can prove that the disease free equilibrium is globally asymptotically stable and attracts the bounded sets of $\mathbb{R} \times L_{+}^{1}(0,+\infty)$. Moreover, when $R_{0}(F)>1$, there is uniform persistence in $\mathbb{R} \times\left(L_{+}^{1}(0,+\infty) \backslash\{0\}\right)$. So by applying Theorem 1.4-4.2, we obtain the following result.

Theorem 4.2 Let Assumption $E$ be satisfied. Then there exists $\varepsilon>0$, such that if $F \in$ $\Lambda,\left\|F-F_{0}\right\|_{\infty} \leq \varepsilon$, and $R_{0}(F)>1$, then the endemic steady state of (4.2) is globally asymptotically stable in $[0,+\infty) \times\left(L_{+}^{1}(0,+\infty) \backslash\{0\}\right)$.

Acknowledgments I would like to first thank Xiaoqiang Zhao and Shigui Ruan for helpful discussions about this work. I also would like to thank the anonymous referee who has substantially contributed to the improvement of this paper.

\section{References}

1. Anita, S.: Analysis and Control of Age-dependent Population Dynamics. Mathematical Modelling: Theory and Applications. Vol. 11, Kluwer Academic Publishers, Dordrecht (2000)

2. Arendt, W., Batty, C.J.K., Hieber, M., Neubrander, F.: Vector-Valued Laplace Transforms and Cauchy Problems. Birkhäuser, Basel (2001)

3. Arino, O.: A survey of structured cell populations. Acta Biotheoretica 43, 3-25 (1995)

4. Babin, A.V., Vishik, M.I.: Attractors of Evolutionary Equations. North-Holland, Amsterdam (1989)

5. Cholewa, J.W., Dlotko, T.: Global Attractors in Abstract Parabolic Problems. Cambridge University Press, Cambridge (2000)

6. Cholewa, J.W., Hale, J.K.: From point dissipativeto compact dissipative- addendum to "some counterexamples in dissipative systems", Dynamics of Continuous. Discr. Impuls. Syst. Ser. A: Math. Anal. 14, 147-164 (2007)

7. D'Agata, E., Magal, P., Ruan, S., Webb, G.F.: Asymptotic behaviorin nosocomial epidemic models with antibiotic resistance. Differ. Integr. Equ. 19, 573-600 (2006)

8. Freedman, H.I., Ruan, S., Tang, M.: Uniform persistence and flowsnear a closed positively invariant set. J. Dyn. Differ. Equ. 6, 583-600 (1994)

9. Gobbino, M., Sardella, M.: On the connectnedness of attractors for dynamical systems. J. Differ. Equ. 133, 1-14 (1997)

10. Grabosch, A.: Compactness properties and asymptotics of strongly coupled systems. J. Math. Anal. Appl. 187, 411-437 (1994)

11. Hale, J.K.: Asymptotic Behavior of Dissipative Systems, Mathematical Surveys and Monographs 25, American Mathematical Society, Providence, RI (1988)

12. Hale, J.K.: Dynamics of numerical approximation. Appl. Math. Comput. 89, 5-15 (1998)

13. Hale J.K.: Dissipation and attractors. In: Fiedler, Groeger and Sprekels (Eds.) International Conference on Differential Equations, (Berlin 1999), World Scientific (2000)

14. Hale, J.K., Raugel, G.: Upper semicontinuity of attractor for singularly perturbed hyperbolic equation. J. Differ. Equ. 73, 197-214 (1988)

15. Hale, J.K., Raugel, G.: Lower semicontinuity of attractors of gradient systems and applications. Annali di Mat Pura Appl. (IV)(CLIV), 281-326 (1989)

16. Hale, J.K., Raugel, G.: Lower semicontinuity of the attractor for a singularly perturbed hyperbolic equation. J. Dyn. Differ. Equ. 2, 19-67 (1900) 
17. Hale, J.K., Raugel, G.: Convergence in gradient-likesystems. ZAMP 43, 63-124 (1992)

18. Hale, J.K., Raugel, G.: Limits of semigroups depending on parameters. Resenhas 1, 1-45 (1993)

19. Hale, J.K., Waltman, P.: Persistence in infinite dimensional systems. SIAM J. Math. Anal. 20, 388395 (1989)

20. Hale, J.K., Lin, X-B., Raugel, G.: Upper semicontinuity of attractors for approximations of semigroups and partial differential equations. Math. Comp. 50, 89-123 (1988)

21. Hirsch, M.W., Smith, H.L., Zhao, X.-Q.: Chain transitivity, attractivity and strong repellors for semi dynamical systems. J. Dyn. Differ. Equ. 13, 107-131 (2001)

22. Hutson, V., Schmitt, K.: Permanence and the dynamics of biological systems. Math. Biosci. 111, 293326 (1992)

23. Iannelli, M.: Mathematical Theory of Age-structured Population Dynamics. Giadini Editori e stampatori in Pisa (1994)

24. Kellermann, H., Hieber, M.: Integrated semigroups. J. Funct. Anal. 84, 160-180 (1989)

25. Lasalle, J.P.: The Stability of Dynamical Systems. SIAM, Philadelphia (1976)

26. Kostin, I.N.: Lower semicontinuity of a non hyperbolic attractor. J. Lond. Math. Soc. II 52, 568$582(1995)$

27. Liu, L., Cohen, J.E.: Equilibrium and local stability in alogistic matrix model for age-structured populations. J. Math. Biol. 25, 73-88 (1987)

28. Magal, P.: A uniqueness result for nontrivial steady stateof a density-dependent population dynamics model. J. Math. Anal. Appl. 233, 148-168 (1999)

29. Magal, P.: A global attractivity result for a discrete time system, with application to a density dependent population dynamics models. Nonlinear Stud. 7, 1-22 (2000)

30. Magal, P.: A global stabilization result for a discrete time dynamical system preserving cone. J. Differ. Equ. Appl. 7, 231-253 (2001)

31. Magal, P.: Compact attractors for time-periodic age structured population models. Electron. J. Differ. Equ. 2001, 1-35 (2001)

32. Magal, P., Ruan, S.: On integrated semigroups and age structured models in $L^{p}$ spaces. Differ. Integr. Equ. 20, 197-139 (2007)

33. Magal, P., Ruan, S.: Center Manifolds for Semilinear Equations with Non-dense Domain and Applications on Hopf Bifurcation in Age Structured Models. Memoirs of the American Mathematical Society (2008) (to appear)

34. Magal, P., Thieme, H.R.: Eventual compactness for a semiflow generated by an age-structured models. Commun. Pure Appl. Anal. 3, 695-727 (2004)

35. Magal, P., Webb, G.F.: Mutation, selection, and recombination in a model of phenotype evolution. Discr. Contin. Dyn. Sys. 6, 221-236 (2000)

36. Magal, P., Zhao, X.-Q.: Global attractors and steady states for uniformly persistent dynamical systems. SIAM J. Math. Anal. 37, 251-275 (2005)

37. Raugel, G.: Dynamics of Partial Differential Equations on Thin Domains. CIME Course, Montecatini Terme, Lecture Notes in Mathematics, vol. 1609, pp. 208-215. Springer (1995)

38. Raugel, G.: Global attractors in partial differential equations, Handbook of Dynamical Systems, vol. 2, pp. 885-982. North-Holland, Amsterdam (2002)

39. Sell, G.R., You, Y.: Dynamics of Evolutionary Equations. Springer, New York (2002)

40. Smith, H.L., Zhao, X.-Q.: Dynamics of a periodically pulsed bio-reactormodel. J. Differ. Equ. 155, 368404 (1999)

41. Smith, H.L., Zhao, X.-Q.: Robust persistence for semidynamical systems. Nonlinear Anal. 47, 61696179 (2001)

42. Smith, H.L., Waltman, P.: Perturbation of a globally stablesteady state. Proc. AMS 127, 447-453 (1999)

43. Stuart, A.M., Humphries, A.R.: Dynamical Systems and Numerical Analysis, Monograph on Applied and Computational Mathematics. Cambridge University Press, Cambridge (1996)

44. Temam, R.: Infinite Dimensional Dynamical Systemsin Mechanics and Physics. Springer, New York (1988)

45. Thieme, H.R.: Semiflows generated by Lipschitz perturbations of non-densely defined operators. Differ. Integr. Equ. 3, 1035-1066 (1990)

46. Thieme, H.R.: Balance exponential growth for perturbed operator semigroups. Adv. Math. Sci. Appl. 10, 775-819 (2000)

47. Thieme, H.R.: Uniform persistence and permanence for non-autonomous semiflows in population biology. Math. Biosci. 166, 173-201 (2000)

48. Thieme, H.R., Castillo-Chavez, C.: On the role of variable infectivity in the dynamics of the human immunodeficiency virus epidemic, in Mathematical and Statistical Approaches to AIDS 
Epidemiology. In: Castillo-Chavez, C. (ed.) Lecture Notes in Biomathematics, vol. 83, pp. 157176. Springer, Berlin, New York (1989)

49. Thieme, H.R., Castillo-Chavez, C.: How mayinfection-age-dependent infectivity affect the dynamics of HIV/AIDS? SIAM J. Appl. Math. 53, 1447-1479 (1993)

50. Vanderbauwhede, A.: Invariant manifold in infinite dimensions. In: Chow, S.N., Hale, J.K. (eds.) Dynamical of Infinite Dimensional Systems, NATO ASI series, F37, pp. 409-420. Springer, New York (1987)

51. Vishik, M.I.: Asymptotic Behavior of Solutions of Evolutionary Equations. Cambridge University Press, Cambridge (1992)

52. Webb, G.F.: Theory of Nonlinear Age-Dependent Population Dynamics. Marcel Dekker (1985)

53. Webb, G.F.: An operator-theoretic exponential growth indifferential equations. Trans. AMS 303, 751763 (1987)

54. Zhao, X.-Q.: Uniform persistence and periodic coexistencestates in infinite-dimensional periodic semiflows with applications. Can. Appl. Math. Q. 3, 473-495 (1995)

55. Zhao, X.-Q.: Dynamical Systems in Population Biology. Springer, New York (2003) 